\title{
GESTÃO ESTRATÉGICA PARA COMPRAS: APLICAÇÃO DA CURVA ABC E MATRIZ KRALJIC DETERMINANDO UM MODELO IDEAL DE PEDIDOS
}

\author{
STRATEGIC PURCHASING MANAGEMENT: APPLICATION OF THE ABC CURVE AND KRALJIC \\ MATRIX DETERMINING AN IDEAL ORDERING MODEL
}

\section{Lucas Matheus Fonseca Bastos ${ }^{1} \&$ Marcos Wagner Jesus Servare Junior ${ }^{2 *}$ \\ ${ }^{12}$ Centro Universitário Salesiano - UniSales \\ ${ }^{1}$ Imatheus01@ gmail.com ${ }^{2 *}$ marcos.servare@ salesiano.br}

\section{ARTIGO INFO.}

\section{Recebido em: 04.11.2021}

Aprovado em: 20.12.2021

Disponibilizado em: 22.12.2021

\section{Palavras-chave:}

Compras; gestão estratégica; planejamento estratégico; análise de estoque; previsão de demanda.

\section{KEYWORDS:}

Purchasing; strategic management; strategic planning; inventory analysis; demand forecast.

*Autor Correspondente: Servare, M. W. J., Junior.

\section{RESUMO}

Diante das dificuldades comerciais vividas entre atacadistas na disputa por preços, parcerias e confiança, para empresas que buscam se destacar é necessário iniciar um trabalho de organização setorial e explorar possibilidades da aplicação de um diferencial competitivo para se sobressair entre os concorrentes diretos. Desta forma, tendo uma boa gestão estratégica a empresa consegue se estabelecer podendo ser assertivo nas tomadas de decisão, seleção de fornecedores e adequação na aquisição de suprimentos. A partir disso, esse estudo tem como objetivo o desenvolvimento de uma condição adequada de reposição de estoque do grupo de produtos "tubulações" cadastrado na empresa, que irão ser classificados com base no nível de importância e impacto financeiro, devendo ajustar as quantidades de aquisição dentro das restrições estabelecidas utilizando ferramentas direcionadas. Sendo elas a Curva $\mathrm{ABC}$, que classifica os principais itens do grupo pelo nível de importância e enquadramento financeiro impactando diretamente na empresa e a Matriz Kraljic que correlaciona os itens com seus riscos de armazenamento e financeiros, direcionando estratégias e auxiliando o comprador na tomada de decisão. Assim desenvolvendo um modelo ideal de pedido mensal, seguindo as restrições estabelecidas e reduzindo os níveis de rupturas no estoque. Também podendo servir como pilar estrutural para novas análises, direcionamentos neste campo e pesquisas futuras.

\begin{abstract}
Given the commercial difficulties experienced between wholesalers in the dispute for prices, partnerships and trust, for companies seeking to stand out, it is necessary to start a sectorial organization work and explore possibilities of applying a competitive differential to stand out among direct competitors. In this way, with good strategic management, the company is able to establish itself and can be assertive in decisionmaking, supplier selection and adequacy in the acquisition of supplies. Based on this, this study aims to develop an adequate stock replenishment condition for the "pipes" product group registered in the company, which will be classified based on the level of importance and financial impact, and should adjust the acquisition quantities within the constraints established using targeted tools. These being the ABC Curve, which classifies the main items of the group by the level of importance and financial framework, directly impacting the company, and the Kraljic Matrix, which correlates the items with their storage and financial risks, directing strategies and assisting the buyer in decision making. Thus, developing an ideal monthly order model, following the established restrictions and reducing the levels of stockouts. It can also serve as a structural pillar for further analysis, directions in this field and future research.
\end{abstract}


Citação (APA): Bastos, L. M. F. \& Servare, M. W. J., Junior. (2021). Gestão estratégica para compras: aplicação da curva abc e matriz Kraljic determinando um modelo ideal de pedidos. Brazilian Journal of Production Engineering, 7(5), 325-341.

\section{INTRODUÇÃO}

No cenário atual a busca por destaque, vantagens competitivas e melhoria contínua vêm crescendo com grande intensidade, visto que esta competitividade cada vez mais acentuada acaba gerando conflitos no mercado de atacadistas, entre as empresas em geral existe uma procura por diferenciação sob a concorrência em termos de flexibilidade, inovação e um padrão de serviços com maior qualidade.

Desta forma para se destacar no mercado a empresa deve se organizar no quesito tomada de decisão, que faz parte da rotina empresarial na gestão estratégica do setor de compras. O encargo fundamental de compras em uma organização é garantir que a aquisição dos itens seja realizada corretamente, na quantidade adequada, com o menor preço, seguindo padrões de negociação e garantindo qualidade no serviço prestado (Slack et al., 1999).

A gestão estratégica no setor de compras é adotada por várias empresas com objetivo de se obter excelência na aquisição de produtos, serviços prestados e seleção adequada de fornecedores, considerando sempre uma boa negociação. Esta seleção adequada de fornecedores tem grande importância entre as organizações, afinal através da utilização de estratégias bem definidas em acordos comerciais pode se obter melhores resultados e vantagens competitivas, além de aumento da eficiência, redução dos custos, rapidez na entrega e melhor preço no mercado, assim se destacando, aumentando o desempenho organizacional.

Diversos realizam a gestão do estoque a partir de diversas ferramentas, tais como o estoque de segurança (Barrow \& Kourentzes, 2016), utilizando tecnologias como RFID (Çakici, Groenevelt \& Seidmann, 2011), técnicas como a programação dinâmica (Fu, Hsu \& Xue, 2017), análise dinâmica de média da variância (Kouvelis, Pang \& Ding, 2018), para controle de diversos itens, como peças sobressalentes (Turrini \& Meissner, 2018).

Esta gestão com estratégias predefinidas e aplicadas corretamente também se torna muito eficaz na redução de faltas e rupturas de estoque, situação que vem atrapalhando o crescimento pontual da empresa referente a um determinado grupo de produtos. Desta forma, a predefinição de estratégias garante que o atacadista sempre tenha produtos a disposição para rápida reposição dos lojistas quando solicitado, sendo assim um diferencial competitivo dentre os concorrentes.

Mediante a dificuldade de atuação no setor este artigo tem como objetivo principal o desenvolvimento de uma condição adequada de reposição de estoque do grupo de produtos "tubulações" cadastrado na empresa, sendo treze produtos ativos (SKU's), que irão ser classificados com base no nível de importância e impacto financeiro, devendo ajustar as quantidades de aquisição dentro das restrições estabelecidas utilizando ferramentas direcionadas. Com essa análise e desenvolvimento adequado, o artigo também poderá servir como fundamento e pilar para novas análises e direcionamentos voltados ao desenvolvimento do setor de compras.

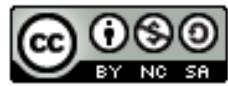


Citação (APA): Bastos, L. M. F. \& Servare, M. W. J., Junior. (2021). Gestão estratégica para compras: aplicação da curva abc e matriz Kraljic determinando um modelo ideal de pedidos. Brazilian Journal of Production Engineering, 7(5), 325-341.

\section{REFERENCIAL TEÓRICO}

A gestão de compras afeta diretamente nos objetivos e capacidades das organizações, com isso, é necessário que os líderes exerçam um bom gerenciamento no setor, pois essa gestão está assumindo cada vez mais o papel estratégico e decisivo nas empresas, pelo fato de ter relacionamento direto com os resultados financeiros (Telles \& Leal, 2019).

O setor de compras exerce responsabilidades em alto nível, existindo uma crescente importância por conta do reconhecimento no volume de gastos direcionados a aquisição de suprimentos na empresa, exigindo ao processo um entendimento e direcionamento correto na abordagem estratégica da atividade (Bowersox, 2006).

Para se exercer com eficiência a tarefa de compras, deve-se planejar e definir os processos que serão executados. Segundo Chiavenato (2004), o "planejamento é a função administrativa que definem objetivos e decide sobre os recursos e tarefas necessárias para alcançá-los adequadamente". Tendo em mãos os processos e tarefas definidas, o setor deverá seguir rigorosamente o determinado para que tenha êxito na atividade.

As aquisições realizadas devem ser moldadas e direcionadas a partir de um estoque máximo e mínimo delimitado pelas demandas existentes. De acordo com Lustosa et al. (2008), "demanda é a disposição dos clientes ao consumo de determinados bens ou serviços de uma organização". Sendo assim, o comprador deve analisar os níveis de estoque e evitar ao máximo os pontos de faltas e rupturas, tendo sempre disponibilidade para atender as solicitações imediatas. Existem dois cenários que devem ser evitados: estoque em excesso, tendo assim duração em longo prazo ou estoque insuficiente e em atraso, gerando perdas, insatisfação e menor produtividade (Chiavenato, 2005).

A escolha de fornecedores, é um processo rigoroso e deve ser analisado detalhadamente para que seja feita uma boa seleção do parceiro distribuidor, existem critérios para seleção deles, de acordo com Baily et al. (2000, p. 352) "Os critérios padronizados para a seleção de fornecedor são: qualidade, preço, condições, entrega e serviço, acompanhados do desempenho real no atendimento de pedidos anteriores". Tendo como objetivo principal nesta avaliação, conseguir a comprovação de que o fornecedor selecionado tenha disponibilidade e capacidade para atender os requisitos específicos quando solicitado, garantindo prazo e qualidade acordado entre as partes interessadas (Gurgel \& Francischini, 2002, p. 62).

\subsection{Técnicas de gestão de estoque}

De forma que seja possível descrever os artigos analisados e apresentar as ferramentas utilizadas e contribuições dadas referente ao tema de pesquisa proposto, será descrito sobre cada artigo do banco selecionado características e crescentes.

Almeida et al. (2020) utilizam estudo de caso a partir de entrevistas semiestruturadas, Análise de atividades e níveis de satisfação como técnicas e contribui com a análise do relacionamento dos fornecedores do tipo contract manufacturer e melhoria do nível aquisitivo e gestão de relacionamento com fornecedores. Desta forma possibilitando direcionamento no quesito tomada de decisões operacionais.

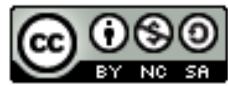



Kraljic determinando um modelo ideal de pedidos. Brazilian Journal of Production Engineering, 7(5), 325-341.

Andrade e Maria (2015) apresenta um Estudo de caso, onde através de pesquisa documental foram levantados dados que posteriormente foram tratados no Excel para análise dos resultados, contribuindo com a melhoria da dinâmica do planejamento estratégico e avaliadas as variáveis em uma planilha direcionada demonstrando os que mais impactaram na execução do planejamento e nos itens de capital em uma instituição de ensino pública.

Aragão, Holanda e Kloeckner (2020) utilizam as ferramentas Curva ABC e a Matriz de Kraljic, auxiliando e contribuindo com a aplicação e demonstração de metodologia no processo de aquisição, utilizando ferramentas que auxiliam na formalização de estratégias para a orientação na efetivação de contratos.

Batalha e Lima (2020) propuseram Modelos de seleção e contratação de fornecedores, softwares de supplier relationship management (SRM) como metodologia e contribuindo diretamente com o auxílio na implementação e análise do Software SEM (Gestão e relacionamento com fornecedores) no setor de compras, e aplicação de modelo de seleção de fornecedores.

Bezerra et al. (2020) utilizaram Método de Análise Hierárquica de Processos (AHP), utilizando dois grupos de insumos como base e análise multicritério de decisão como ferramentas para contribuir com a obtenção e seleção dos indicadores mais importantes para dar suporte à tomada de decisão e na verificação da técnica utilizada sendo adaptável ao departamento aplicado possibilitando ajustes e modificações.

Bonato et al. (2019) utilizam a metodologia de pesquisa-ação, com isso, o estudo de caso deste projeto utilizando a pesquisa-ação possibilita a verificação na literatura de métodos e cases de desenvolvimento de fornecedores, identificada a melhor ferramenta para ser utilizada.

Borba et al. (2015) se basearam em uma metodologia cálculos, análises e sistema de conformidades para contribuir e alcançar o objetivo de realizar a aplicação do sistema máximo-mínimo no controle de estoques a partir do sistema de demandas, assim auxiliando realizar compras com exatidão evitando rupturas e faltas de produtos em estoque.

Carvalho e Alves (2020) utilizaram as ferramentas Six Sigma, do DMAIC e do PDCA, Brainstormings, Gráficos de Pareto, 5W2H, PPAPs, Follow-Ups, para contribuir com a aplicação das ferramentas da qualidade na busca de melhorias no setor de compras, melhoria contínua, benefícios e capacidades, garantindo direcionamento em negociações e decisões com a aplicação das ferramentas.

Esteves et al. (2020) utilizam o Analytic Hierarchy Process (AHP) na priorização de tipos de compra para realizar a aplicação de uma gestão eficaz no setor de compras, impulsionando a melhoria aquisitiva e análise de priorização dos tipos de compras, cada produto possui seu nível de giro, com isso alguns necessitam de priorização na gestão de compras.

Ganga et al. (2016) propõem uma Revisão sistemática da literatura, a lógica Fuzzy nas técnicas multicritério, técnicas de análise de conteúdo via tag clouds e de análise de correspondência, buscando a iniciativa de coletar, identificar, selecionar e avaliar criticamente

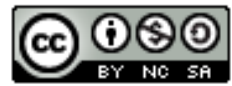


os métodos quantitativos utilizados na seleção de fornecedores sustentáveis do ponto de vista verde e social.

Machado, Guimarães e Lara (2020) realizam um estudo de caso com uma abordagem qualitativa buscando esclarecer e desenvolver uma melhoria na rede de suprimentos, fazendo a ligação direta com fornecedores, transportadora, distribuidor, revendedor e cliente final. Impulsionando fatores relacionados a melhoria de custos e aquisições.

Merces, Pacheco e Oliveira (2020) utilizaram a ferramenta Curva ABC para contribuir com avaliação da curva que cada item e fornecedor se enquadram, possibilitando traçar uma estratégia e redução de custos. Cada item possuindo sua classificação facilita no momento de aplicação de forças e estratégias sejam de compra ou venda.

Moraes et al. (2020) fizeram uso de uma técnica e metodologia a utilização de Pesquisa Operacional, modelagem matemática, cálculos dos erros de previsão (MAE, RMSE e MAPE), Aditivo de Winters, Contribuindo na procura de métodos para previsão de demanda que tenha maior acurácia e aprimorar o planejamento de recursos. Utilizando a pesquisa operacional com modelos matemáticos, estatísticos buscando a redução de desperdícios ou superlotação de estoque e previsões.

Pimentel, Cruz e Guerreiro (2016) utilizam a média ponderada para previsões de curto prazo e o 4MAD, contribuindo com aprovação de metodologias e mostra a importância da previsão de demanda para vendas futuras dos produtos da empresa, a utilização da média ponderada auxilia no entendimento e aperfeiçoamento das previsões e demandas direcionadas.

Ronchi et al. (2016) usaram a Curva ABC, estoques máximos e mínimos e a rotatividade do estoque como metodologia buscando uma melhoria na gestão e auxiliando na análise de estoque máximo utilizando ferramentas disponíveis, na redução do nível elevado de estoque maximizando a rotatividade. Possibilitando na classificação dos produtos distribuindo forças no momento de realizar ações e aproveitamento nas compras.

Santos e Silva (2020) utilizam Holt-Winters, softwares Microsoft Excel e Minitab possibilitando a visualização e contribuição para resolução de problemas com previsão de demanda. A utilização de planilhas e holt-winters funcionam como algoritmo, direcionando a gestão e possibilitando noções de demanda e necessidades aquisitivas de forma organizada.

Saraiva, Pereira e Souza (2015) realizam levantamentos de dados, utilizando ERP e programação de suprimentos para atingir os objetivos traçados e contribui na melhoria da gestão dos serviços desenvolvidos pelos setores da empresa, financeiro e poder aquisitivo. De certa forma, programando a aquisição de suprimentos com o ERP, facilita na gestão e análise de dados, voltados a indústria salineira.

Silva et al. (2020) tem como ferramenta o Sistema MRP de Planejamentos na aplicação de um estoque de segurança, a disponibilidade e qualidade na produção, redução do lead time do ciclo produtivo. Calculando de forma assertiva desde a aquisição da matéria prima, os processos produtivos até o produto acabado e entregue.

Toleto et al. (2020) com o Mapeamento de processo BPMN, possibilita a visualização de tomada de decisão e na definição de qual modal de importação melhor se encaixa nas 

Kraljic determinando um modelo ideal de pedidos. Brazilian Journal of Production Engineering, 7(5), 325-341.

situações. Mapeando os processos facilita a detecção de falhas reduzindo perdas, direcionando a escolha de um modal de importação adequado para a empresa listada.

Finalizando com Viana et al. (2020) realizam uma abordagem quantitativa e ferramentas de validação da previsão de demanda, visando melhorias e contribuindo com o entendimento de capacidade produtiva necessária em cada período, auxilia na compra de suprimentos e na promoção de uma melhor administração dos estoques. No caso da empresa a pronta entrega, deve sempre ter produtos disponíveis, evitando ao máximo rupturas, pois trazer prejuízos a organização.

\subsection{Curva ABC}

De acordo com a competitividade do mercado a gestão do atacado necessita de selecionar e caracterizar os produtos para distribuição correta das forças nas compras e vendas seja para identificar pontos altos na hora de investir ou até mesmo por solicitação de fornecedores, deve-se realizar e divisão das curvas dos produtos disponíveis em estoque.

Assim, garantindo que as compras sejam feitas da maneira correta. A metodologia da curva $\mathrm{ABC}$ de compras dispõe da seguinte divisão:

- Itens de classe A: de maior prioridade, possuem alto valor e a maior porcentagem do dinheiro investido no estoque, $20 \%$ desses itens correspondem a $80 \%$ do valor;

- Itens de classe B: ainda relevantes possuindo parte significante no valor total de estoque, $30 \%$ desses itens correspondem a $15 \%$ do valor;

- Itens de classe C: de menor importância e menor investimento, 50\% desses itens correspondem a $5 \%$ do valor.

Podendo haver variações na quantidade de itens por classificação, considerando o direcionamento da empresa, caso opte em trabalhar com maior quantidade de itens de Curva A em seu portfólio cadastrado ou uma variação escalando entre A, B e C.

A elaboração da curva $\mathrm{ABC}$ será auxiliada pela análise de relatórios de estoque, compras e vendas por mark-up referente ao período selecionado, emitido pelo comprador da empresa.

Após a emissão e análise do relatório com os itens e informações listados, deve-se atribuir classificação decrescente para cada item, de maneira que o $1^{\circ}$ seja o item de maior volume de compra e venda. Já o último classificado sendo o de menor giro. Perante a elaboração dessa classificação, com base no valor e quantidade total será realizada a somatória acumulada dos percentuais. Seguindo este critério quantitativo, os grupos A, B e C, serão divididos de acordo com a classificação adequada dos produtos.

\subsection{Matriz Kraljic}

A Matriz Kraljic, também conhecida como Matriz de Compras, é bastante utilizada no gerenciamento dos processos, com a implementação de um check-up estratégico de todo portifólio de itens cadastrados entre fornecedores e compradores, possibilita a definição de abordagens direcionadas de forma adequada no setor de compras. A matriz Kraljic busca oferecer base referencial no processo de compras, definindo preferencias e estratégias de compras, produtos selecionados por categorias e características, otimizando a realização de análises entre custos e riscos, capacitando a escolha dos produtos e sua aquisição em duas 
esferas: Capacidade de pagamento e Risco de abastecimento (Kraljic, 1983). Sendo necessário adequar a classificação Kraljic e seus quadrantes, pode-se observar nos Quadro 1 e Quadro 2, juntamente com os significados de cada classe.

Quadro 1. Classificação Kraljic e seus significados.

\begin{tabular}{|c|c|}
\hline Classificação Kraljic & Significado \\
\hline Item não crítico & Fácil de comprar, Baixo impacto financeiro, Produto exposição. \\
\hline Item de alavancagem & Fácil de comprar, Alto impacto financeiro, Produto exposição. \\
\hline Item de gargalo & Dificuldade logística, Baixo impacto financeiro, Produto Especial. \\
\hline Item estratégico & Alta dependência, Alto impacto financeiro, Produto Crucial. \\
\hline
\end{tabular}

Fonte: Autores (2021).

Quadro 2. Quadrantes Kraljic, significados e foco principal para resolução.

\begin{tabular}{|c|c|c|}
\hline Quadrante & O que significa? & Foco \\
\hline Item não crítico & $\begin{array}{c}\text { Fácil de comprar, Baixo impacto financeiro, } \\
\text { Produto exposição. }\end{array}$ & $\begin{array}{c}\text { Aprimorar processos de } \\
\text { compras }\end{array}$ \\
\hline Item de alavancagem & $\begin{array}{c}\text { Fácil de comprar, Alto impacto financeiro, } \\
\text { Produto exposição. }\end{array}$ & Explorar poder de compras \\
\hline Item de gargalo & $\begin{array}{c}\text { Dificuldade logística, Baixo impacto financeiro, } \\
\text { Produto Especial. }\end{array}$ & Minimizar Risco \\
\hline Item estratégico & $\begin{array}{c}\text { Alta dependência, Alto impacto financeiro, } \\
\text { Produto Crucial. }\end{array}$ & $\begin{array}{c}\text { Manter parcerias } \\
\text { estratégicas }\end{array}$ \\
\hline
\end{tabular}

Fonte: Autores (2021).

\section{DEFINIÇÃO DO PROBLEMA}

Atualmente no mercado atacadista a busca por destaque e vantagens competitivas vem aumentando o nível de exigências no quesito tomada de decisão, tendo de ser realizado de forma mais ágil, pela alta necessidade de reposição de acordo com os níveis críticos de estoque e pela variedade de itens e fornecedores cadastrados.

Dentro desse cenário o setor de compras assume um papel fundamental de gestão estratégica de suprimentos na organização. Neste sentido, analisando a situação desta empresa atacadista que atualmente passa por dificuldades na reposição de estoque de um grupo de produtos, tendo faltas indesejadas repentinas de tubos no galpão, tem-se como principal objetivo a busca por uma condição adequada de reposição de estoque do grupo de tubulações ativos, contendo treze produtos cadastrados (SKU's), que devem ser classificados de acordo com seu nível de importância tendo que ajustar as quantidades dentro das restrições estabelecidas, contribuindo com a organização e controle de cada produto, considerando que o espaço físico do galpão suporta 5.000 unidades de tubos que devem ser dividias adequadamente entre os treze produtos para que não exista durante o decorrer do mês rupturas de estoque e também que o contrário não ocorra, gerando uma superlotação.

Tendo também como restrição a regra implantada pelo fornecedor para compras, classificando os produtos no pedido, impedindo o atacado comprar apenas curva $\mathrm{A}$, sendo necessário conter no pedido ao menos $15 \%$ de curva B e C. Referente ao tempo de entrega do fornecedor, são de três dias úteis após o momento da compra, considerando que podem acontecer vendas fora da curva, tendo assim a necessidade de acompanhamento diário do comprador, pelo fato do espaço físico ser restrito e se tratar de um atacado com função de rápido reabastecimento não

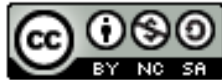


podendo ficar sem produtos para atender os clientes. Com isso, é necessário classificar os produtos do grupo que será trabalhado, utilizando as ferramentas Curva $\mathrm{ABC}$ de produtos, para designar suas classes, referente a importância e impacto quantitativo e financeiro, auxiliando na gestão dos produtos.

A Matriz Kraljic utilizada para segmentar os produtos selecionados dividindo-os em quatro classes de acordo com suas complexidades sejam, itens de alavancagem, itens estratégicos, itens não críticos e gargalos, buscando a otimização da relação entre riscos dos produtos e custos, seguido pela classificação de relevância dos itens. Podendo desta forma ser realizada uma gestão estratégica de compras de tubulações, tendo definida por final uma quantidade adequada a ser comprada de cada produto para suprir as necessidades de vendas mensais, atendendo de forma eficaz as restrições existentes.

\section{IMPLEMENTAÇÃO DE GESTÃO DE ESTOQUE}

Considerando as diretrizes de classificação e as restrições exigidas pela empresa, inicialmente foi realizada a seleção dos itens e o levantamento de dados para desenvolver (Quadro 3 e Tabela 1).

Quadro 3. Seleção inicial dos itens do Grupo tubulações.

\begin{tabular}{ll}
\hline COD. & DESCRICAO DO ITEM \\
\hline 00662 & TUBO ESGOTO SERIE NORMAL 100MM \\
00664 & TUBO ESGOTO SERIE NORMAL 150MM \\
00665 & TUBO ESGOTO SERIE NORMAL 200MM \\
00658 & TUBO ESGOTO SERIE NORMAL 40MM \\
00659 & TUBO ESGOTO SERIE NORMAL 50MM \\
00661 & TUBO ESGOTO SERIE NORMAL 75MM \\
00654 & TUBO SOLDAVEL DE 20MM \\
00655 & TUBO SOLDAVEL DE 25MM \\
00656 & TUBO SOLDAVEL DE 32MM \\
01596 & TUBO SOLDAVEL DE 40MM \\
00657 & TUBO SOLDAVEL DE 50MM \\
01622 & TUBO SOLDAVEL DE 60MM \\
05115 & TUBO SOLDAVEL DE 75MM \\
\hline
\end{tabular}

Fonte: Autores (2021).

Tabela 1. Vendas mensais dos itens selecionados e média de vendas.

\begin{tabular}{llrrrr}
\multicolumn{5}{c}{ (VENDAS MENSAIS) } \\
\hline COD. & DESCRICAO DO ITEM & $06 / 2021$ & $07 / 2021$ & $08 / 2021$ & MEDIA \\
\hline 00662 & TUBO ESGOTO SERIE NORMAL 100MM & $2.048,00$ & $1.988,00$ & $2.257,00$ & $2.097,67$ \\
00664 & TUBO ESGOTO SERIE NORMAL 150MM & 122,00 & 86,00 & 166,00 & 124,67 \\
00665 & TUBO ESGOTO SERIE NORMAL 200MM & 80,00 & 39,00 & 49,00 & 56,00 \\
00658 & TUBO ESGOTO SERIE NORMAL 40MM & 887,00 & 909,00 & $1.008,00$ & 934,67 \\
00659 & TUBO ESGOTO SERIE NORMAL 50MM & 969,00 & 945,00 & $1.103,00$ & $1.005,67$ \\
00661 & TUBO ESGOTO SERIE NORMAL 75MM & 486,00 & 389,00 & 379,00 & 418,00 \\
00654 & TUBO SOLDAVEL DE 20MM & $2.612,00$ & $2.708,00$ & $2.860,00$ & $2.726,67$ \\
00655 & TUBO SOLDAVEL DE 25MM & $2.820,00$ & $3.142,00$ & $3.787,00$ & $3.249,67$ \\
00656 & TUBO SOLDAVEL DE 32MM & 210,00 & 360,00 & 320,00 & 296,67 \\
01596 & TUBO SOLDAVEL DE 40MM & 41,00 & 19,00 & 41,00 & 33,67 \\
00657 & TUBO SOLDAVEL DE 50MM & 371,00 & 418,00 & 424,00 & 404,33 \\
01622 & TUBO SOLDAVEL DE 60MM & 30,00 & 22,00 & 23,00 & 25,00 \\
05115 & TUBO SOLDAVEL DE 75MM & 0,00 & 5,00 & 14,00 & 6,33 \\
\hline
\end{tabular}

Fonte: Autores (2021). 
Após a seleção dos itens do grupo e o levantamento de dados referente as vendas mensais, o preço de cada produto foi listado podendo assim desenvolver a Curva ABC dos produtos (Tabela 2).

Tabela 2. Curva ABC de produtos.

\begin{tabular}{|c|c|c|c|c|c|c|c|c|}
\hline Código & Descrição do item (SKU) & Valor Unit. (R\$) & $\begin{array}{c}\text { Venda Média } \\
\text { Mensal }\end{array}$ & Valor total & $\begin{array}{c}\text { Porcentagem } \\
\text { Individual }\end{array}$ & $\begin{array}{c}\text { Porcentagem } \\
\text { Acumulada }\end{array}$ & Classificação & \multirow{8}{*}{ - $46 \%$ SKU (79,72\% RECETA) } \\
\hline 00662 & TUBO ESGOTO SERIE NORMAL 100MM & $R \$ 52,08$ & 2.098 & $R \$ 109.246,65$ & $33,17 \%$ & $33,17 \%$ & A & \\
\hline 00655 & TUBO SOLDAVEL DE 25MM & $\mathrm{R} \$ 13,66$ & 3.250 & $\mathrm{R} \$ 44.390,49$ & $13,48 \%$ & $46,65 \%$ & A & \\
\hline 00659 & TUBO ESGOTO SERIE NORMAL 5OMM & $\mathrm{R} \$ 35,29$ & 1.006 & $\mathrm{R} \$ 35.490,09$ & $10,78 \%$ & $57,43 \%$ & A & \\
\hline 00654 & TUBO SOLDAVEL DE 20MM & $R \$ 10,88$ & 2.727 & $\mathrm{R} \$ 29.666,17$ & $9,01 \%$ & $66,44 \%$ & A & \\
\hline 00657 & TUBO SOLDAVEL DE 50MM & $R \$ 58,21$ & 404 & $R \$ 23.536,05$ & $7,15 \%$ & $73,58 \%$ & A & \\
\hline 00661 & TUBO ESGOTO SERIE NORMAL 75MM & $\mathrm{R} \$ 48,38$ & 418 & $\mathrm{R} \$ 20.222,84$ & $6,14 \%$ & $79,72 \%$ & A & \\
\hline 00664 & TUBO ESGOTO SERIE NORMAL 150MM & $R \$ 153,25$ & 125 & $\mathrm{R} \$ 19.105,68$ & $5,80 \%$ & $85,52 \%$ & B & \\
\hline 00658 & TUBO ESGOTO SERIE NORMAL 4OMM & $\mathrm{R} \$ 20,16$ & 935 & $\mathrm{R} \$ 18.842,95$ & $5,72 \%$ & $91,25 \%$ & B & \multirow[t]{6}{*}{ - 23\% SKU (16,32\% RECEIA $)$} \\
\hline 00665 & TUBO ESGOTO SERIE NORMAL 200MM & $\mathrm{R} \$ 282,34$ & 56 & $\mathrm{R} \$ 15.811,04$ & $4,80 \%$ & $96,05 \%$ & B & \\
\hline 00656 & TUBO SOLDAVEL DE 32MM & $\mathrm{R} \$ 28,30$ & 297 & $\mathrm{R} \$ \mathbf{8 . 3 9 5 , 7 6}$ & $2,55 \%$ & $98,60 \%$ & C & \\
\hline 01622 & TUBO SOLDAVEL DE 60MM & $\mathrm{R} \$ 86,51$ & 25 & $\mathrm{R} \$ 2.162,75$ & $0,66 \%$ & $99,25 \%$ & c & \\
\hline 01596 & TUBO SOLDAVEL DE 40MM & $\mathrm{R} \$ 44,82$ & 34 & $\mathrm{R} \$ 1.509,09$ & $0,46 \%$ & $99,71 \%$ & c & \\
\hline 05115 & TUBO SOLDAVEL DE 75MM & $R \$ 150,73$ & 6 & $\mathrm{R} \$ 954,12$ & $0,29 \%$ & $100,00 \%$ & C & \\
\hline & & & Total & $\mathrm{R} \$ 329.333,69$ & & & & \\
\hline
\end{tabular}

Fonte: Autores (2021).

Observações da empresa e Curva ABC desenvolvida:

- Itens de Curva A, são trabalhados com margem de venda inferior (Menos lucro, maior giro).

- Por se tratar de uma empresa atacadista, realmente os produtos de Curva A são mais visados, pois são os de extremo giro (Mais comprados e mais vendidos). Logo, por opção da empresa foi escolhido ter cadastrado no leque de produtos mais itens de alto giro.

- A classificação dos produtos referente a curva é realizada a partir do percentual de valor agregado de cada item, se aproximando as determinações das curvas: A 80\% / B $15 \%$ / C $5 \%$.

Desta forma, após a realização da Curva $\mathrm{ABC}$, foi desenvolvido um diagrama de Pareto, possibilitando destacar o nível dos produtos de acordo com suas classes, considerando suas porcentagens individuais e porcentagens acumuladas (Imagem 1).

Imagem 1. Diagrama de Pareto.

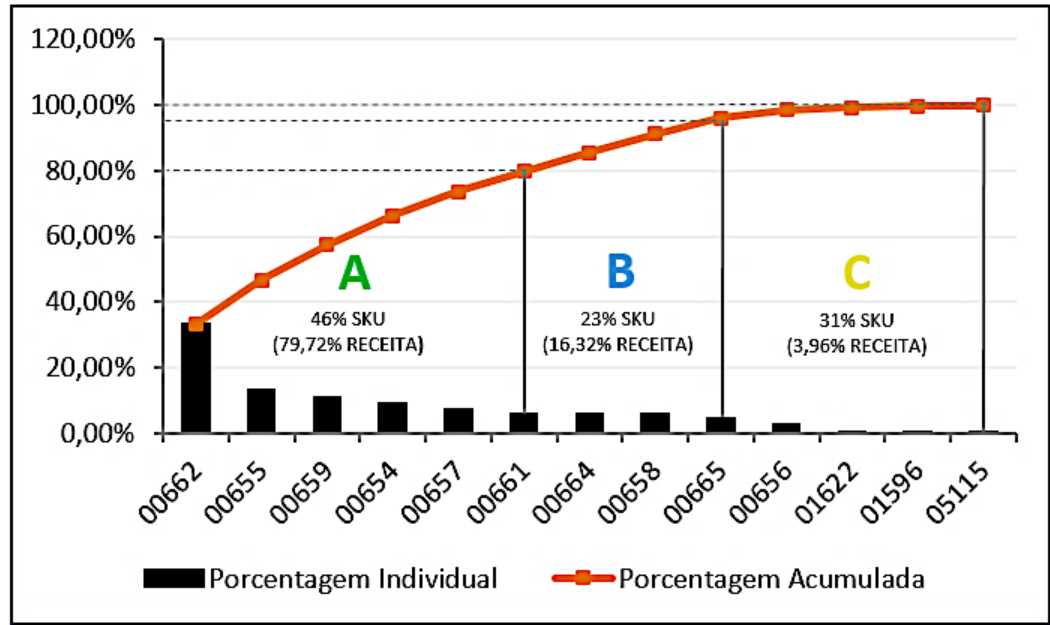

Fonte: Autores (2021).

Tendo descrito os tópicos e quadrantes para classificação, foi listado no Quadro 1 os itens e classificar de acordo com o risco de fornecimento e impacto no lucro, possibilitando aplicar o modelo Kraljic nos produtos.

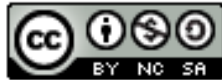


Citação (APA): Bastos, L. M. F. \& Servare, M. W. J., Junior. (2021). Gestão estratégica para compras: aplicação da curva abc e matriz Kraljic determinando um modelo ideal de pedidos. Brazilian Journal of Production Engineering, 7(5), 325-341.

Quadro 1. Classificação dos itens referente a riscos de fornecimento e lucro.

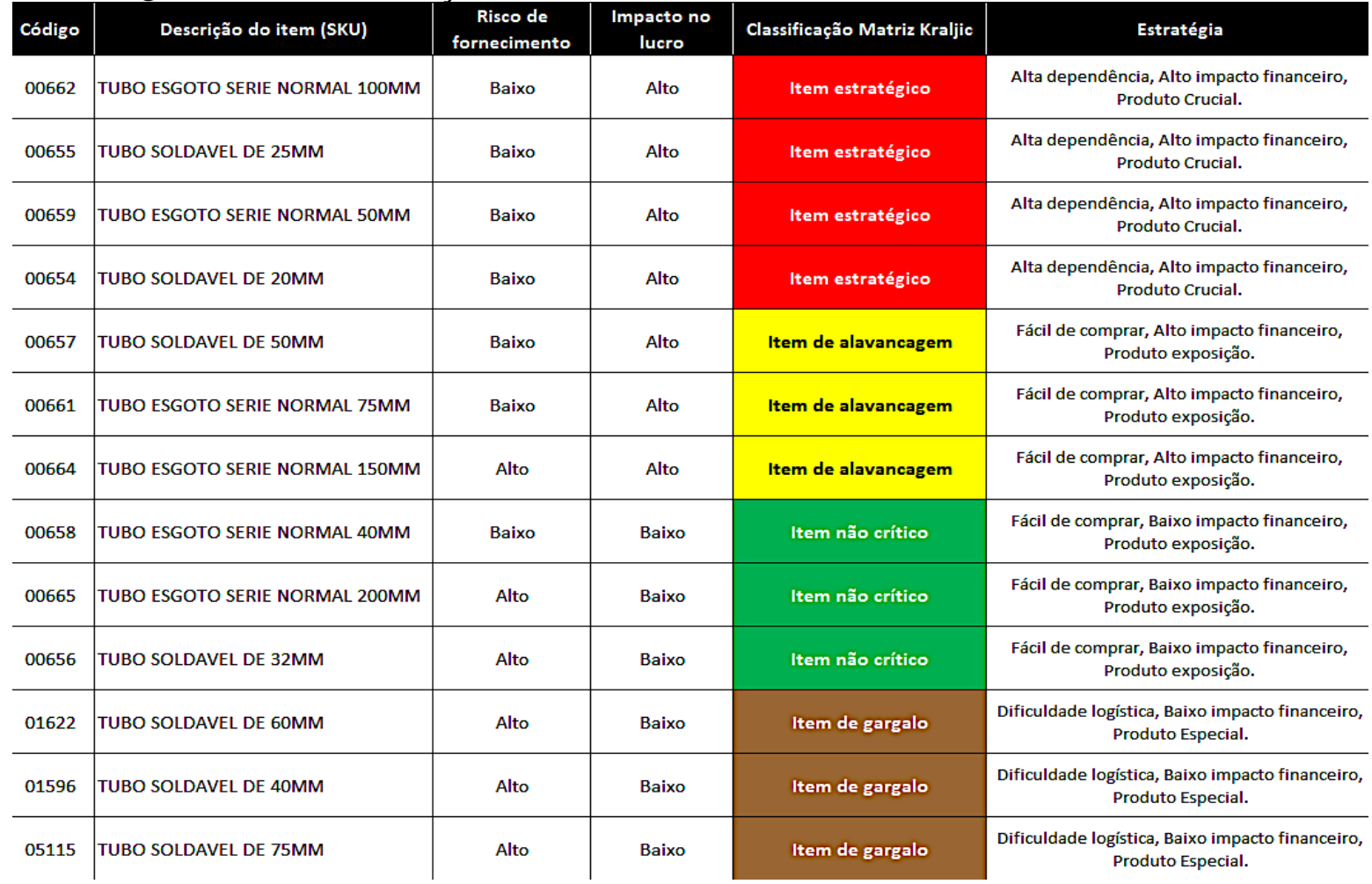

Fonte: Autores (2021).

Classificando os itens de acordo com o risco de fornecimento, impacto no lucro e quadrantes de Kraljic, temos os grupos formados e direcionados a estratégia de cada um, possibilitando assim o entendimento das ações a serem realizadas. Nos itens estratégicos, deve-se manter a parceria com o fornecedor de forma que exista a melhor condição e reposição possível. Nos itens de alavancagem o foco principal é explorar o poder de compra e também fortificar a parceria com o fornecedor.

Nos itens não críticos, por serem itens de fácil reposição, logística e financeira, deve-se aprimorar os processos de compra, facilitando ainda mais as atividades para uma aquisição adequada. Nos itens de gargalo, por serem os mais críticos do grupo, necessitam de uma atenção maior na aquisição, transporte e armazenagem, tomando medidas preventivas para reduzir riscos (Imagem 2).

Imagem 2. Matriz Kraljic do grupo de produtos

MATRIZ DE KRALJIC
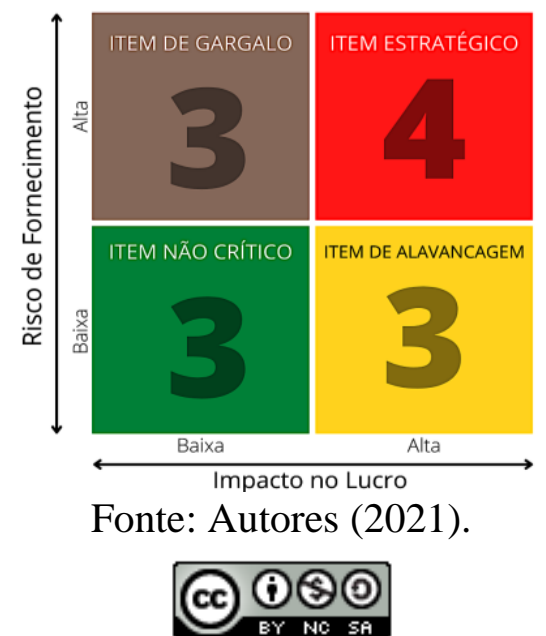

Esta obra está licenciada com uma Licença Creative Commons Atribuição-Não Comercial-Compartilha Igual 4.0 Internacional. Brazilian Journal of Production Engineering, São Mateus, Editora UFES/CEUNES/DETEC. 
Citação (APA): Bastos, L. M. F. \& Servare, M. W. J., Junior. (2021). Gestão estratégica para compras: aplicação da curva abc e matriz Kraljic determinando um modelo ideal de pedidos. Brazilian Journal of Production Engineering, 7(5), 325-341.

\subsection{Relevância dos itens e suas características}

De acordo com as duas ferramentas utilizadas, Curva ABC e Matriz Kraljic, foi possível realizar uma classificação dos itens cadastrados no grupo. Com isso, essa classificação é apresentada no Quadro 2 considerando as informações levantadas, mostrando a relevância de cada item e suas características, assim direcionando uma ideia de necessidade de compras, para elaborar a simulação de compra quinzenal adequada quando solicitado.

Quadro 2. Relevância dos itens e suas características.

\begin{tabular}{|c|c|c|c|}
\hline Descrição do item & $\begin{array}{c}\text { Classificação } \\
\text { ABC }\end{array}$ & Classificação Kraljic & Características \\
\hline TUBO ESGOTO SERIE NORMAL 100MM & \multirow{6}{*}{$\mathbf{A}$} & Item estratégico & \multirow{4}{*}{$\begin{array}{l}\text { Produtos de altíssimo giro, } \\
\text { cliente busca preço e rápida } \\
\text { reposição, com isso deve-se } \\
\text { manter parcerias estratégicas } \\
\text { com os fornecedores. Tendo } \\
\text { maior parte do estoque no galpão } \\
\text { e as maiores vendas diárias. }\end{array}$} \\
\hline TUBO SOLDAVEL DE 25MM & & Item estratégico & \\
\hline TUBO ESGOTO SERIE NORMAL 5OMM & & Item estratégico & \\
\hline TUBO SOLDAVEL DE 20MM & & Item estratégico & \\
\hline TUBO SOLDAVEL DE 5OMM & & Item de alavancagem & \multirow{3}{*}{$\begin{array}{l}\text { Produtos com bom nível de } \\
\text { venda, possui facilidade de } \\
\text { reposição e agrega } \\
\text { financeiramente bem, deve-se } \\
\text { explorar o poder de compra. } \\
\text { Estoque mediano no galpão. }\end{array}$} \\
\hline TUBO ESGOTO SERIE NORMAL 75MM & & Item de alavancagem & \\
\hline TUBO ESGOTO SERIE NORMAL 150MM & \multirow{3}{*}{ B } & Item de alavancagem & \\
\hline TUBO ESGOTO SERIE NORMAL 4OMM & & Item não crítico & \multirow{3}{*}{$\begin{array}{c}\text { Produtos fáceis de comprar, } \\
\text { porém com baixo impacto } \\
\text { financeiro. É necessário atenção } \\
\text { na compra para não ultrapassar o } \\
\text { nível máximo de estoque e } \\
\text { sempre buscar aprimorar os } \\
\text { processos de compra. }\end{array}$} \\
\hline TUBO ESGOTO SERIE NORMAL 200MM & & Item não crítico & \\
\hline TUBO SOLDAVEL DE 32MM & \multirow{4}{*}{$\mathbf{C}$} & Item não crítico & \\
\hline TUBO SOLDAVEL DE 60MM & & Item de gargalo & \multirow{3}{*}{$\begin{array}{l}\text { Produtos mais críticos do grupo, } \\
\text { havendo necessidade de muita } \\
\text { atenção na compra, possuindo } \\
\text { baixíssimo giro, geralmente } \\
\text { apenas em vendas casadas, por } \\
\text { encomenda. }\end{array}$} \\
\hline TUBO SOLDAVEL DE 40MM & & Item de gargalo & \\
\hline TUBO SOLDAVEL DE 75MM & & Item de gargalo & \\
\hline
\end{tabular}

Fonte: Autores (2021).

Com as duas ferramentas aplicadas, mesmo não tendo relação entre ambas, foi realizado uma junção entre as classificações ACB e os quatro quadrantes Kraljic, possibilitando verificar quantos Itens estratégicos, Itens de alavancagem, Itens não críticos ou Itens de gargalo, existem em cada classificação $\mathrm{A}, \mathrm{B}$ e $\mathrm{C}$, e de acordo com o mercado descrever as características de cada classificação, desta forma, pode-se observar que na classificação A, a empresa possui quatro Itens estratégicos e dois Itens de alavancagem, na classificação B, um Item de alavancagem e dois Itens não críticos e já na classificação $\mathrm{C}$, contamos com um Item não crítico e três Itens de gargalo. Como listados, os Itens estratégicos são itens de altíssimo giro, ocupando maior parte do estoque de tubos no galpão e maior número de vendas diárias, Os itens de alavancagem, são itens com bom nível de vendas e ocupa um espaço considerável no galpão. Itens não críticos são produtos fácies de comprar, porém tendo de ter atenção nas compras para não exceder o estoque máximo. Finalizando nos Itens de gargalo são itens

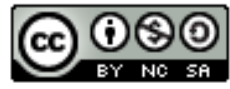


específicos, mais críticos, possuindo baixíssimo giro e grande parte solicitados por encomenda.

\section{ANÁLISE dOS RESULTADOS}

O principal fator a ser analisado neste estudo foi à carteira de compras da empresa atacadista de materiais para construção no Espírito Santo, com ênfase no grupo de tubulações, possuindo 13 produtos cadastrados no grupo, com o intuito de classificar os produtos de acordo com seu nível de venda e auxiliar no direcionamento de compra e tomada de decisão reduzindo faltas ou superlotação de estoque, cumprindo a risca as restrições de armazenamento.

Com a aplicação da Curva ABC (Tabela 1) foi possível verificar que por se tratar de uma empresa atacadista, os produtos do portfólio por sua maioria são classificados como alto giro "Curva A", itens com demanda elevada, seguindo por itens de Curva B e C com níveis de vendas inferiores, mas que de certa forma são necessários para complementar o mix de produtos do parceiro fornecedor e elevando a rentabilidade da empresa.

A partir do desenvolvimento da Matriz Kraljic foi possível definir as preferencias e estratégias de compras para formular as simulações mensais, produtos selecionados por categorias e características, buscando aperfeiçoar a realização de análises entre os custos e riscos de cada item do grupo de tubulações, capacitando o comprador e direcionando a aquisição em duas esferas: Capacidade de pagamento e Risco de abastecimento. Com isso possibilitando o entendimento das ações a serem realizadas e facilitando a divisão de quantidades a serem compradas nos pedidos distribuídos no decorrer do mês. Após aplicação das duas ferramentas foi realizada uma classificação dos itens unindo dados coletados e listagem de informações e características sobre cada produto.

Desta forma, com os direcionamentos após as classificações foram desenvolvidos três modelos de pedidos de compra, sendo distribuídos como Pedido 1, Pedido 2 e Pedido 3. Seguindo as condições de armazenamento com máxima de 5000 tubos no galpão e considerando o prazo de três dias úteis para entrega dos produtos pelo fornecedor.

Apresentado Tabela 3, o pedido 1 será realizado no início de cada mês, como modelo de introdução foi o pedido de maior volume, relacionando a quantidade total de 5000 tubos iniciais e valor total de $\mathrm{R} \$ 155.177,20$.

Tabela 3. Pedido de compra 1.

\begin{tabular}{|c|c|c|c|c|}
\hline Código & Descrição do ttem & Qtd. Comprar & Vr. Unitário & Valor Total $\nabla$ \\
\hline 00662 & TUBO ESGOTO SERIE NORMAL 100MM & $1.000,0000$ & 52,0800 & $52.080,00$ \\
\hline 00655 & TUBO SOLDAVEL DE 25MMM & $1.400,0000$ & 13,6600 & $19.124,00$ \\
\hline 00659 & TUBO ESGOTO SERIE NORIMAL 5OMM & 400,0000 & 35,2900 & $14.116,00$ \\
\hline 00654 & TUBO SOLDAVEL DE $20 \mathrm{MM}$ & $1.200,0000$ & 10,8800 & $13.056,00$ \\
\hline 00657 & TUBO SOLDAVEL DE 5OMM & 195,0000 & 58,2100 & $11.350,95$ \\
\hline 00661 & TUBO ESGOTO SERIE NORMAL 75MM & 195,0000 & 48,3800 & $9.434,10$ \\
\hline 00664 & TUBO ESGOTO SERIE NORMAL 15OMM & 60,0000 & 153,2500 & $9.195,00$ \\
\hline 00658 & TUBO ESGOTO SERIE NORIMAL 40MM & 395,0000 & 20,1600 & $7.963,20$ \\
\hline 00665 & TUBO ESGOTO SERIE NORMAL 200MM & 20,0000 & 282,3400 & $5.646,80$ \\
\hline 00656 & TUBO SOLDAVEL DE 32MM & 110,0000 & 28,3000 & $3.113,00$ \\
\hline 01622 & TUBO SOLDAVEL DE 60MM & 10,0000 & 86,5100 & 865,10 \\
\hline 01596 & TUBO SOLDAVEL DE $40 \mathrm{MM}$ & 12,0000 & 44,8200 & 537,84 \\
\hline 05115 & TUBO SOLDAVEL DE $75 M M$ & 3,0000 & 150,7300 & 452,19 \\
\hline & & & VR.TOTAL: & $155.177,20$ \\
\hline
\end{tabular}

Fonte: Autores (2021). 
Citação (APA): Bastos, L. M. F. \& Servare, M. W. J., Junior. (2021). Gestão estratégica para compras: aplicação da curva abc e matriz Kraljic determinando um modelo ideal de pedidos. Brazilian Journal of Production Engineering, 7(5), 325-341.

Este pedido possui 5000 unidades de tubos, distribuídos corretamente de acordo com o indicado nas vendas médias mensais. Sendo o maior pedido dentre os realizados, já direcionando aos próximos apenas reposições de quantidades que não puderam ser compradas neste. Em seguida, o pedido 2 (Veja Tabela 4) será implantado uma semana após o pedido 1, com intuito de suprir as quantidades não compradas no primeiro pedido, assim, evitando rupturas e mantendo o estoque abastecido após este período de uma semana de vendas. $\mathrm{O}$ pedido 2 complementa a compra anterior e tem quantidades e valores reduzidas, contendo um total de 3190 unidades e valor total de $\mathrm{R} \$ 96.291,10$.

Tabela 4. Pedido de compra 2.

Código Descrição do Htem

Qtd. Comprar Vr. Unitário Valor Total $\nabla$

\begin{tabular}{|c|c|c|c|c|}
\hline 00662 & TUBO ESGOTO SERIE NORIMAL 100MM & 549,0000 & 52,0800 & $28.591,92$ \\
\hline 00655 & TUBO SOLDAVEL DE $25 \mathrm{MM}$ & 925,0000 & 13,6600 & $12.635,50$ \\
\hline 00659 & TUBO ESGOTO SERIE NORIMAL 5OMM & 303,0000 & 35,2900 & $10.692,87$ \\
\hline 00654 & TUBO SOLDAVEL DE 20IMIM & 764,0000 & 10,8800 & $8.312,32$ \\
\hline 00657 & TUBO SOLDAVEL DE $50 \mathrm{MM}$ & 105,0000 & 58,2100 & $6.112,05$ \\
\hline 00658 & TUBO ESGOTO SERIE NORIMAL 40MM & 270,0000 & 20,1600 & $5.443,20$ \\
\hline 00661 & TUBO ESGOTO SERIE NORIMAL 75MM & 111,0000 & 48,3800 & $5.370,18$ \\
\hline 00665 & TUBO ESGOTO SERIE NORIMAL 200MM & 18,0000 & 282,3400 & $5.082,12$ \\
\hline 00664 & TUBO ESGOTO SERIE NORIMAL $150 M M$ & 32,0000 & 153,2500 & $4.904,00$ \\
\hline 00656 & TUBO SOLDAVEL DE 32MM & 93,0000 & 28,3000 & $2.631,90$ \\
\hline 01622 & TUBO SOLDAVEL DE 60MM & 7,0000 & 86,5100 & 605,57 \\
\hline 01596 & TUBO SOLDAVEL DE 40MM & 11,0000 & 44,8200 & 493,02 \\
\hline 05115 & TUBO SOLDAVEL DE 75MM & 2,0000 & 150,7300 & 301,46 \\
\hline & & & VR.TOTAL: & $96.291,10$ \\
\hline
\end{tabular}

Fonte: Autores (2021).

Caminhando para finalizar as compras do mês do grupo de tubulações sem exceder o máximo estoque disponível e sem rupturas de mercadorias, o terceiro pedido será implantado uma semana após o Pedido 2. Assim se aproximando da metade do mês e apenas cumprindo o fechamento acompanhando os níveis de vendas, evitando situações críticas em caso de vendas fora da curva, que caso venha a acontecer o comprador possui flexibilidade para repor eventuais faltas.

Desta forma, o Pedido 3 (Tabela 5) possui nível de quantidades e valor total muito aproximado ao Pedido 2, por serem compras de reposição, com média de vendas já levantadas anteriormente. Tendo 3193 unidades de tubos e valor total de $\mathrm{R} \$ 96.466,12$.

Tabela 5. Pedido de compra 3

\begin{tabular}{|c|c|c|c|c|}
\hline Código & Descrição do Item & Qtd. Comprar & Vr. Unitário & Valor Total $\nabla$ \\
\hline 00662 & TUBO ESGOTO SERIE NORIMAL $100 \mathrm{MM}$ & 549,0000 & 52,0800 & $28.591,92$ \\
\hline 00655 & TUBO SOLDAVEL DE 25MM & 925,0000 & 13,6600 & $12.635,50$ \\
\hline 00659 & TUBO ESGOTO SERIE NORMAL 50MM & 303,0000 & 35,2900 & $10.692,87$ \\
\hline 00654 & TUBO SOLDAVEL DE 20MM & 764,0000 & 10,8800 & $8.312,32$ \\
\hline 00657 & TUBO SOLDAVEL DE 5OMM & 105,0000 & 58,2100 & $6.112,05$ \\
\hline 00658 & TUBO ESGOTO SERIE NORIMAL 40MM & 270,0000 & 20,1600 & $5.443,20$ \\
\hline 00661 & TUBO ESGOTO SERIE NORIMAL 75MM & 112,0000 & 48,3800 & $5.418,56$ \\
\hline 00665 & TUBO ESGOTO SERIE NORIMAL 200MM & 18,0000 & 282,3400 & $5.082,12$ \\
\hline 00664 & TUBO ESGOTO SERIE NORIMAL 150MM & 33,0000 & 153,2500 & $5.057,25$ \\
\hline 00656 & TUBO SOLDAVEL DE 32MM & 94,0000 & 28,3000 & $2.660,20$ \\
\hline 01622 & TUBO SOLDAVEL DE 60MM & 8,0000 & 86,5100 & 692,08 \\
\hline 01596 & TUBO SOLDAVEL DE 40MM & 11,0000 & 44,8200 & 493,02 \\
\hline 05115 & TUBO SOLDAVEL DE $75 \mathrm{MM}$ & 1,0000 & 150,7300 & 150,73 \\
\hline & & & VR.TOTAL: & $96.466,12$ \\
\hline
\end{tabular}

Fonte: Autores (2021). 

Kraljic determinando um modelo ideal de pedidos. Brazilian Journal of Production Engineering, 7(5), 325-341.

Assim, tendo estoque disponível para suprir a venda mensal, sem extrapolar o nível de estoque máximo delimitado. Podendo sofrer pequenos ajustes acima ou abaixo dos modelos. Frisando que os pedidos acima já possuem em suas quantidades um adicional buscando suprir os três dias de entrega do fornecedor para a próxima compra, evitando que neste meio tempo de faturamento, expedição e entrega falte itens para venda, conforme observado no Quadro 3.

Quadro 3. Cronograma de compra e duração de pedidos.

\begin{tabular}{|c|c|c|c|c|}
\hline \multicolumn{7}{|c|}{ CRONOGRAMA DE DURAÇÃO DOS TRÊS PEDIDOS NO MÊS. } \\
\hline \# PEDIDO & $1^{\circ}$ SEMANA & $2^{\circ}$ SEMANA & $3^{\circ}$ SEMANA & $4^{\circ}$ SEMANA \\
\hline 1 & 1 & 1 & & \\
\hline 2 & & Implanta Pedido 2 & 2 & \\
\hline 3 & & & Implanta Pedido 3 & 3 \\
\hline
\end{tabular}

Fonte: Autores (2021).

\section{CONSIDERAÇÕES FINAIS}

O presente artigo foi desenvolvido sob um detalhado estudo com levantamentos de dados e análises direcionadas a um grupo de produtos de uma empresa atacadista para materiais de construção, considerando o nível de venda de cada produto, preço de compra, classificação detalhada, tomada de decisão, viabilidade de aquisição, riscos de armazenagem e financeiros, seguindo à risca as restrições da empresa, para isso é necessário conhecimento de ferramentas para realizar um gerenciamento ideal no setor de compras. Entre estas ferramentas a Curva ABC e Matriz de Kraljic, possibilitando identificar os itens de maior importância e classificar produtos no portifólio de acordo com as necessidades pontuais.

Ao aplicar os conceitos da Curva $\mathrm{ABC}$ no grupo de tubulações da empresa atacadista, foi possível verificar e analisar a importância e o enquadramento financeiro que cada produto que compõe esse grupo impacta na organização. Permitindo assim identificar os itens de maior importância e maior giro, possibilitando classificá-los, determinando quais precisam de maior atenção no momento da compra. Foi observado que $46 \%$ dos SKU's do grupo pertencem a Curva A, impactando em $79,72 \%$ da receita, já a Curva B com $23 \%$ dos produtos, impactando com 16,32\% e a Curva C com 31\% dos SKU's impactando financeiramente 3,96\%. Assim foi possível levantar que, a empresa possui mais produtos de alto giro cadastrados (Curva A), mas também complementa seu estoque com baixo nível nas curvas B e C.

Após isso, aplicando a Matriz de Kraljic foi possível identificar o quadrante de cada produto, seja item não crítico, item de alavancagem, item de gargalo e item estratégico, relacionandoos entre riscos financeiros e riscos de armazenagem, aplicando uma estratégia direcionada para cada um deles. Sobre os quadrantes, os itens estratégicos, o comprador deve estabelecer uma parceria com o fornecedor garantindo que exista a melhor condição possível entre preço e rápida reposição. Nos itens de alavancagem o comprador deve explorar o poder de compra e fortificar a parceria com o fornecedor. Nos itens não críticos, por serem itens de fácil reposição, logística e financeira, deve-se aprimorar os processos de compra, facilitando as atividades para uma aquisição adequada e nos itens de gargalo, por serem os mais críticos do grupo, necessitam de uma atenção maior do comprador no momento da aquisição e acompanhamento no transporte e armazenagem, tomando medidas preventivas para reduzir

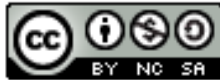


riscos. Assim determinando que o grupo possui 3 itens não críticos, 3 itens de alavancagem, 3 itens de gargalo e 4 itens estratégicos.

Após utilização das ferramentas e análises realizadas, foi desenvolvido um modelo de pedido de compra mensal para a empresa, se dividindo em três partes. Sendo o primeiro pedido de estocagem, armazenando o estoque máximo possível, pedido gerado para vendas de duas semanas, mesmo sendo abaixo do nível de venda, mas considerando o fato da restrição de 5000 unidades de tubos em estoque e o prazo de entrega de três dias do fornecedor. Em sequência o segundo pedido deverá ser implantado uma semana após o Pedido 1, assim tendo tempo de baixar o nível de estoque e reponto com este segundo pedido que irá completar a baixa e suprirá mais uma semana de venda. E o terceiro pedido que será implantado uma semana após o Pedido 2, cumprindo o fechamento do mês acompanhando os níveis de vendas, evitando situações críticas em caso de vendas fora da curva, em tais situações o comprador possui flexibilidade para repor eventuais faltas.

Finalmente podemos concluir que com o uso da Curva ABC e Matriz de Kraljic na análise de estoque da empresa atacadista, foi possível desenvolver uma modelo de compra real e ideal, garantindo que não faltem produtos para venda e nem exceda o nível máximo de estoque, possuindo estratégias pré-definidas evitando surpresas e problemas posteriores, alcançando o objetivo determinado.

Para pesquisas futuras, sugere-se este artigo como fundamento e pilar em novas análises e direcionamentos voltados a gestão, controle e desenvolvimento do setor de compras, auxiliando na aplicação das ferramentas utilizadas.

\section{REFERÊNCIAS}

Almeida, V. M. R., et al. (2020). Gestão dos fornecedores de embalagens e uma empresa de cosméticos. Anais do Encontro Nacional de Engenharia de Produção, Foz do Iguaçu, PR, Brasil, 40 .

Andrade, J. I., de. \& Maria, R. C. (2015). Planejamento Estratégico no setor público: análise das variáveis que influenciam o processo de compras em uma instituição de ensino. Anais do Encontro Nacional de Engenharia de Produção, Fortaleza, CE, Brasil, 35.

Aragão, L. M. X., Holanda, T. C., \& Kloeckner, N. V. R. (2020). Estratégias no setor de compras que influenciam a performance das aquisições de materiais indiretos em uma empresa alimentícia. Anais do Encontro Nacional de Engenharia de Produção, Foz do Iguaçu, PR, Brasil, 40.

Baily, P., Farmer, D., Jessop, D., \& Jones, D. (2000). Compras: Princípios e Administração. São Paulo: Atlas 182p.

Barrow, D. K., \& Kourentzes, N. (2016). Distributions of forecasting errors of forecast combinations: implications for inventory management. International Journal of Production Economics, 177, 24-33.

Batalha, A. A. \& Lima, Renato S. (2020). Gestão de relacionamento com fornecedores em uma empresa do setor de bens de consumo: um estudo de caso. Anais do Encontro Nacional de Engenharia de Produção, Foz do Iguaçu, PR, Brasil, 40.

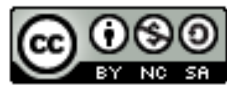


Citação (APA): Bastos, L. M. F. \& Servare, M. W. J., Junior. (2021). Gestão estratégica para compras: aplicação da curva abc e matriz Kraljic determinando um modelo ideal de pedidos. Brazilian Journal of Production Engineering, 7(5), 325-341.

Bezerra, R. R. R., et al. (2020). Proposta de metodologia para avaliação e seleção de fornecedores em uma indústria madeireira: Uma abordagem baseada em Análise Hierárquica de Processos. Anais do Encontro Nacional de Engenharia de Produção, Foz do Iguaçu, PR, Brasil, 40.

Bonato, S. V., et al. (2019). Iniciativas de desenvolvimento de fornecedores: estudo de caso em uma indústria de equipamentos de limpeza. Anais do Simpósio de Engenharia de Produção, Bauru, SP, Brasil, 26.

Borba, J. C. R., et al. (2015). Aplicação do sistema máximo-mínimo no controle de estoque de uma empresa do segmento termoplástico. . Anais do Encontro Nacional de Engenharia de Produção, Fortaleza, CE, Brasil, 35.

Bowersox, Donald J., et al. (2006). Gestão Logística de Cadeia de Suprimentos. 1 ed. Bookman: Porto Alegre.

Çakıcı, Ö. E., Groenevelt, H., \& Seidmann, A. (2011). Using RFID for the management of pharmaceutical inventory-system optimization and shrinkage control. Decision Support Systems, 51(4), 842-852.

Carvalho, G. B. \& Alves, N. A. (2020). Aplicação das Ferramentas da Qualidade no Setor de Compras Visando Redução de Custos. Anais do Encontro Nacional de Engenharia de Produção, Foz do Iguaçu, PR, Brasil, 40.

Chiavenato, I. (2005). Administração da produção: uma abordagem introdutória. Rio de Janeiro: Elsevier.

Chiavenato, I. (2004). Administração nos Novos Tempos. 2. ed. Rio de Janeiro: Elsevier.

Esteves, R. F. G., et al. (2019). Priorização de tipos de compra por critérios comerciais e aplicação de AHP. Anais do Simpósio de Engenharia de Produção, Bauru, SP, Brasil, 27.

Fu, K., Gong, X., Hsu, V. N., \& Xue, J. (2021). Dynamic Inventory Management with Inventory-based Financing. Production and Operations Management.

Ganga, G. M. D., et al. (2016). Métodos quantitativos para seleção de fornecedores sustentáveis: uma revisão sistemática da literatura. Revista Produção Online, 16(4), 14341457.

Gurgel, F. A. \& Francischini, P. (2002). Administração de Materiais e do Patrimônio. São Paulo: Pioneira.

Kraljic, P. (1983). Purchasing must become supply management. Harvard business review, 61(5), 109-117.

Kouvelis, P., Pang, Z., \& Ding, Q. (2018). Integrated commodity inventory management and financial hedging: A dynamic mean-variance analysis. Production and Operations Management, 27(6), 1052-1073.

Lustosa, L. J., Mesquita, M. A. de., Quelhas, O. L. G., \& Oliveira, R. J., de. (2008). Planejamento e Controle da Produção. 2 ed. Rio de Janeiro: Elsevier.

Machado, S. L.; Guimarães, M. R. N.; \& Lara, F. F. (2020). A importância das compras de indiretos e suas estratégias em uma empresa de bens de consumo. Anais do Simpósio de Engenharia de Produção, Bauru, SP, Brasil, 27.

Merces, R. M., Pacheco, B. C. S., \& Oliveira, L. M. (2020). O uso da curva abc para gestão de estoques e fornecedores: uma aplicação em uma indústria de compressores do interior de

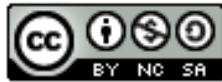


Citação (APA): Bastos, L. M. F. \& Servare, M. W. J., Junior. (2021). Gestão estratégica para compras: aplicação da curva abc e matriz Kraljic determinando um modelo ideal de pedidos. Brazilian Journal of Production Engineering, 7(5), 325-341.

São Paulo. Anais do Encontro Nacional de Engenharia de Produção, Foz do Iguaçu, PR, Brasil, 40.

Moraes, N. T., et al. (2020). Cálculo e comparação de modelos de previsão de demanda de uma fabricante de componentes plásticos. Anais do Encontro Nacional de Engenharia de Produção, Foz do Iguaçu, PR, Brasil, 40.

Pimentel, F. Q. A., Cruz, R. G. G., \& Guerreiro, R. P. (2016). Aplicação de técnicas de previsão de demanda em uma loja de materiais de construção. Anais do Encontro Nacional de Engenharia de Produção, João Pessoa, PB, Brasil, 36.

Ronchi, L. M., et al. (2016). Os reflexos da análise da curva ABC na gestão de estoques de uma empresa comercial de materiais de construção. Anais do Encontro Nacional de Engenharia de Produção, João Pessoa, PB, Brasil, 36.

Santos, T. da S., Silva, \& L. M. F. (2020). Aplicação do método de previsão de demanda holtwinters para uma empresa de bebidas. Anais do Encontro Nacional de Engenharia de Produção, Foz do Iguaçu, PR, Brasil, 40.

Saraiva, A. C. C., Pereira, J. R., \& De Sousa, J. A. (2015). Programação de suprimentos e decisões de compras: o impacto em uma indústria salineira. Anais do Encontro Nacional de Engenharia de Produção, Fortaleza, CE, Brasil, 35.

Silva, M. F. da., et al. (2020). Gestão de estoque com a implantação do sistema MRP (Planejamento de Requerimento de Materiais) em uma indústria de reatores. Anais do Encontro Nacional de Engenharia de Produção, Foz do Iguaçu, PR, Brasil, 40.

Slack, N., Chamber, S., Hardland, C., Harrison, A., \& Johnston, R. (1999). Administração da Produção. Ed. Atlas, São Paulo.

Telles, L. S. \& Leal, R. (2019). A gestão de compras em uma empresa do setor de comércio e serviços: utilizando o método do custo padrão na homologação de fornecedores. Revista Contexto, 19(41), 74-89.

Toledo, S. M. M. et al. (2020). Proposta de Aplicação da Teoria da Utilidade para Tomada de Decisão: Escolha do Modal de Importação em um Processo de Compras numa Empresa do Setor Tecnológico. Anais do Encontro Nacional de Engenharia de Produção, Foz do Iguaçu, PR, Brasil, 40.

Turrini, L., \& Meissner, J. (2019). Spare parts inventory management: New evidence from distribution fitting. European Journal of Operational Research, 273(1), 118-130.

Viana, D. de A. et al. (2020). Estudo comparativo entre modelos de previsão de demanda em uma empresa de pronta entrega. Anais do Encontro Nacional de Engenharia de Produção, Foz do Iguaçu, PR, Brasil, 40. 